\title{
Polyacylurethanes as Novel Degradable Cell Carrier Materials for Tissue Engineering
}

\author{
Danijela Jovanovic ${ }^{1}$, Frans V. Roukes ${ }^{1}$, Andrea Löber ${ }^{2}$, Gerwin E. Engels ${ }^{3}$, \\ Willem van Oeveren ${ }^{3}$, Xavier J. Gallego van Seijen ${ }^{4}$, Marja J.A. van Luyn ${ }^{4}$, \\ Martin C. Harmsen ${ }^{4}$ and Arend Jan Schouten ${ }^{1, *}$
}

1 Department of Polymer Science, Faculty of Mathematics and Natural Sciences, Zernike Institute for Advanced Materials, University of Groningen, Nijenborgh 4, 9747 AG Groningen, The Netherlands; E-Mails: d.jovanovic@rug.nl (D.J.); frans_roukes@hotmail.com (F.V.R.)

2 Institute for Bioengineering, Martin-Luther University, Halle 06108, Germany; E-Mail: andrea_loeber@gmx.de

3 Department of Biomedical Engineering, University Medical Centre Groningen, University of Groningen, A. Deusinglaan 1, building 3215, FB40, 9713AV Groningen, The Netherlands; E-Mails: g.e.engels@med.umcg.nl (G.E.E.);w.van.Oeveren@med.umcg.nl (W.O.)

4 Department of Pathology and Medical Biology, University Medical Centre Groningen, University of Groningen, Hanzeplein 1, 9713 GZ, Groningen, The Netherlands; E-Mails: x_gallego@hotmail.com (X.J.G.S.); m.j.a.van.Luyn@med.umcg.nl (M.J.A.L.); m.c.harmsen@med.umcg.nl (M.C.H.)

* Author to whom correspondence should be addressed; E-Mail: a.j.schouten@rug.nl; Tel.: +31-50-363-4513; Fax: +31-50-363-4400.

Received: 10 September 2011 / Accepted: 21 September 2011 / Published: 6 October 2011

\begin{abstract}
Polycaprolactone (PCL) polyester and segmented aliphatic polyester urethanes based on PCL soft segment have been thoroughly investigated as biodegradable scaffolds for tissue engineering. Although proven beneficial as long term implants, these materials degrade very slowly and are therefore not suitable in applications in which scaffold support is needed for a shorter time. A recently developed class of polyacylurethanes (PAUs) is expected to fulfill such requirements. Our aim was to assess in vitro the degradation of PAUs and evaluate their suitability as temporary scaffold materials to support soft tissue repair. With both a mass loss of $2.5-3.0 \%$ and a decrease in molar mass of approx. 35\% over a period of 80 days, PAUs were shown to degrade via both bulk and surface erosion mechanisms. Fourier Transform Infra Red (FTIR) spectroscopy was successfully applied to study the extent of PAUs microphase separation during in vitro degradation. The
\end{abstract}


microphase separated morphology of PAU1000 (molar mass of the oligocaprolactone soft segment $=1000 \mathrm{~g} / \mathrm{mol}$ ) provided this polymer with mechano-physical characteristics that would render it a suitable material for constructs and devices. PAU1000 exhibited excellent haemocompatibility in vitro. In addition, PAU1000 supported both adhesion and proliferation of vascular endothelial cells and this could be further enhanced by pre-coating of PAU1000 with fibronectin (Fn). The contact angle of PAU1000 decreased both with in vitro degradation and by incubation in biological fluids. In endothelial cell culture medium the contact angle reached $60^{\circ}$, which is optimal for cell adhesion. Taken together, these results support the application of PAU1000 in the field of soft tissue repair as a temporary degradable scaffold.

Keywords: biodegradable polymers; tissue engineering; biomedical polyurethanes; hydrolytic degradation; acylurethanes; blood compatibility; endothelial cells

\section{Introduction}

Degradable polymers are preferred candidates for designing therapeutic devices to treat missing or damaged soft tissues. Being FDA approved, polycaprolactone (PCL) has been intensively investigated as temporary scaffold biomaterial. However, PCL suffers from significant drawbacks. PCL is found to degrade very slowly both in vitro and in vivo, with almost no mass loss or decrease in molar mass for at least 6 months of degradation [1,2]. In addition, in order to achieve good mechanical properties, the molar mass of PCL has to be relatively high which leads to an increase in crystalline fraction of this semi-crystalline polyester. The latter might cause an obstacle for healthy regeneration in vivo $[3,4]$.

Our aim was to develop a biomaterial with tunable (degradation) properties and surface characteristics that allow for cell adhesion to serve as temporary support in soft tissue regeneration.

Polyurethanes possess good mechanical properties and blood compatibility, which have made them attractive for their use for manufacture of biomedical devices [5]. The possibility to alter their mechanical properties by changing the ratio between the constituent components, so-called soft segments (polyether, polyester, and polycarbonate) and hard segments (aliphatic or aromatic diisocyanates), renders them very useful in a variety of materials with different requirements.

To the best of our knowledge Endo et al. [6] have described polyacylurethanes in literature for the first time in 1994. The polymers were prepared by the polyaddition of bis(N-acyl isocyanates) with low molecular weight diols and polyether diols. The most interesting feature of this polymerization was the high reactivity under mild conditions of the bis( $\mathrm{N}$-acyl isocyanates) towards the polyether diols without the use of additional catalysts.

In a patent of 1995 Yabuta and Urano claimed the preparation of polyacylurethanes where the diol could be a polycaprolactone prepolymer, introducing this class of polymers in the area of thermoplastic elastomers [7]. However, the procedure described involved quite high temperatures and long reaction times and resulted "preferably" in rather low molar mass polymers. Apparently also preferred was the use of self-condensing unsymmetrical monomers containing only one acylisocyanate group. The polymers have been characterized as easy-degradable. 
Recently, polyacylurethanes (PAUs) have been developed in our laboratory [8,9]. These materials were produced without the use of any toxic catalysts of which remnants might limit biomedical use. Polyacylurethanes are synthesized with terephthaloyl diisocyanate (TPHDI), which is expected not only to enhance materials mechanical properties due to improved micro-phase separation [10], but is also expected to result in non-toxic degradation products [11]. The acyl functionality contributes to the greater reactivity of TPHDI [12] and is expected to hydrolyze relatively fast [13].

The goal of this research was to assess the hydrolytical degradation of PAUs, with an accent on the mechanism of PAU hydrolysis and microphase separation, and to evaluate these materials for application in regenerative medicine as a biodegradable scaffold. Describing the degradation of PAUs in vitro will be used as a starting point to explain its in vivo behavior in the future.

In this study, we show the results of the degradation study of PAUs with different lengths of the oligo( $\varepsilon$-caprolactone) soft segments (Number average molar mass $=1000,1500$ and $2000 \mathrm{~g} / \mathrm{mol}$, PDI $=1.69,1.82$ and 1.90, respectively). PAU characterization included monitoring mass loss, molar masses, and thermal and surface properties of PAUs upon a degradation period of 80 days. The microphase separation of PAUs was analyzed by utilizing Fourier Transform Infra Red (FTIR) spectroscopy. PAU1000 (molar mass of the oligocaprolactone soft segment $=1000 \mathrm{~g} / \mathrm{mol}$ ) was selected as the potential scaffold material to be used in regenerative medicine due to its optimal behavior during hydrolytic degradation and suitable micro-phase separation behavior. On this particular PAU composition, a series of biological assays were performed to test cytotoxicity and haemocompatibility. Human umbilical vein derived endothelial cells (HUVEC) were used for in vitro cell adhesion and proliferation experiments. Since both cell adhesion and blood interactions with biomaterials take place in the material-protein (cell) interface [14,15], surface properties of PAU1000 were also investigated.

\section{Results and Discussion}

\subsection{Mass Loss and Molar Mass Change}

Polyester urethanes are believed to undergo hydrolytic degradation via two different mechanisms identified as bulk degradation and surface erosion [16,17]. Bulk degradation is characterized by an overall decrease of molar mass if random chain scission is occurring. Surface erosion is the process where hydrolysis removes only polymeric chains from the outer layer of the material and this leaves the bulk of the material untouched [16]. Surface erosion is favored for many applications of polymeric biomaterials (e.g., controlled drug delivery), because the material properties remain virtually intact since degradation proceeds through removal of very thin layers of the material.

For all three PAUs, mass loss showed a similar pattern, with a 2.5-3.0 wt $\%$ decrease in mass at the final time point (Figure 1), which is relatively high, especially when compared to other PCL-based materials that are intended for similar applications and which show almost no mass loss even for more than a year $[1,18]$. The apparent high mass loss at the first incubation time point (5 days) can be attributed to different sample manipulation of the incubation samples compared to the non-incubated samples. During preparations for the degradation study (see Section 3.4) prior to weighing, samples were exposed to air and thus may have absorbed water. Therefore, by calculating the linear fit, we only 
compared the mass loss trends of the three PAUs and calculated the mass loss at the end of the examination period (day 80). Since the potential impurities and residual non-reacted material were removed by means of Soxhlet extraction with n-hexane, the observed mass loss upon incubation can only be ascribed to PAU degradation. The PAU films changed from flexible to brittle and fragile with the course of degradation (Figure 1).

Figure 1. Mass loss of polyacylurethane (PAU) films with different soft segment lengths at $37^{\circ} \mathrm{C}$ in phosphate buffer ( $\left.\mathrm{pH}=7.2\right)$. (०) PAU1000; ( $)$ PAU1500 and ( $\boldsymbol{\nabla}$ ) PAU2000.

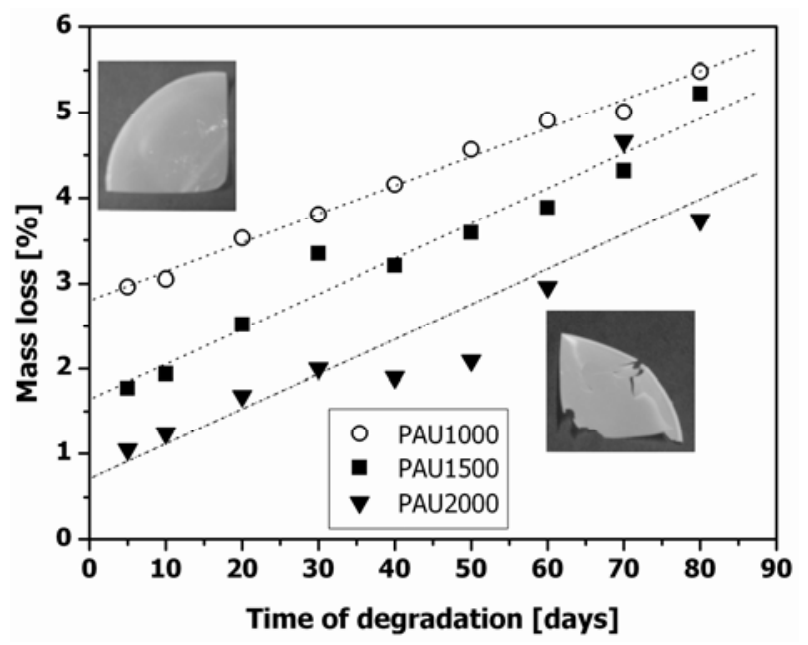

The degradation was accompanied by molar mass decrease, indicating that chain scission in bulk had occurred additionally. The molar mass of degraded PAUs decreased approximately $32 \%$ for PAU1000 and PAU1500, to 38\% for PAU2000 compared to the non-degraded samples (data not shown). It is generally believed that in vitro degradation of polyester urethanes proceeds via random scission of the ester bond of the polyester soft segment. Pitt et al. [19] derived the following equation to describe the $M_{n}$ decrease during ester hydrolysis:

$$
\frac{1}{M_{n}}=\frac{1}{M_{n}{ }^{0}} \times e^{k \times t}
$$

or in its linearized form:

$$
\ln \left(M_{n}\right)=\ln \left(M_{n}^{0}\right)-k \times t
$$

where $M_{n}\left[\mathrm{gmol}^{-1}\right]$ is the number average molar mass of the polymer at any time point, $M_{n}{ }^{0}$ is the initial number average molar mass $\left[\mathrm{g} \mathrm{mol}^{-1}\right], k$ is the ester hydrolysis rate constant $\left[\right.$ day $\left.{ }^{-1}\right]$ and $t$ is the degradation time [day]. If we assume that the above described model is applicable for PAU degradation, then values of $\ln \left(M_{n}{ }^{0}\right)$ of PAUs as a function of $t$ can be described by a linear function (Figure 2).

However, when fit parameters were analyzed, $r^{2}$ value for PAU1000 and PAU1500 fit were $\sim 0.6$ which indicated a poor fit. This further implied that the PAU degradation proceeded not only via scission of ester bonds of the PCL soft segment. Water can attack the following functional groups: Ester groups of the soft segments, acyl groups of the hard segments and urethane groups of the hard segments (Figure 3). As a result, aliphatic carboxylic acids, aromatic carboxylic acids, aliphatic 
alcohols and primary amides can be formed. Both hydrolysis of the soft segment esters and acyl urethane groups are acid catalyzed and expected to contribute greatly to PAU degradation.

Figure 2. Decrease of PAUs' molar mass in the course of hydrolytic degradation. (a) PAU1000 compared to PCL1000-PU; (b) PAU1500 compared to PCL1600-PU; (c) PAU2000 compared to PCL2300-PU. Data set for PCL-PUs was calculated assuming the same $M_{n}{ }^{0}$ and oligodiol molar mass of corresponding PAUs and hydrolysis rate constant $(k)$ value for Polycaprolactone (PCL)-PUs as given by Heijkants et al. [18].

A

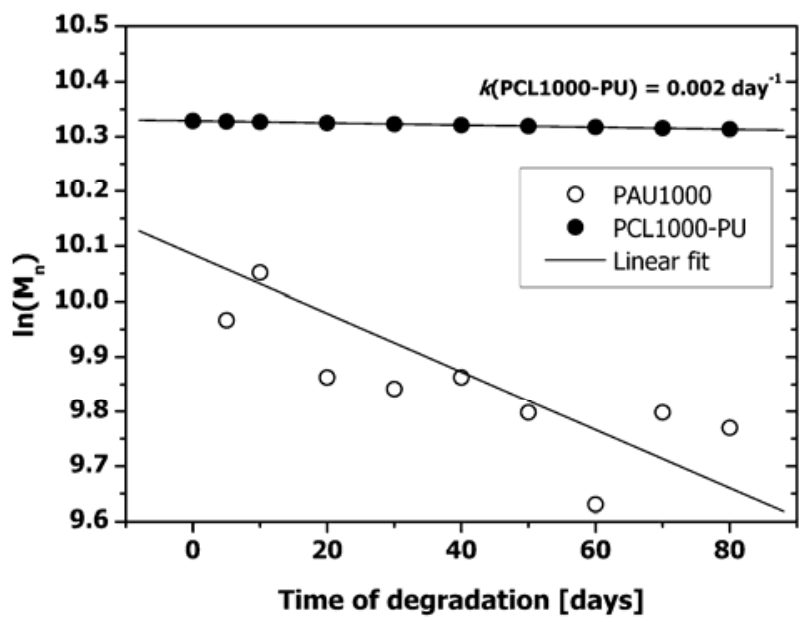

B

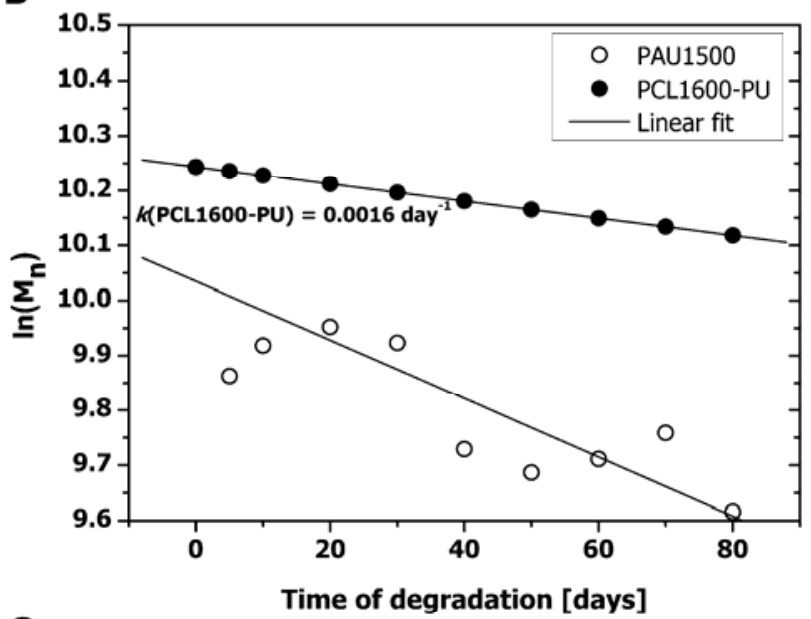

C

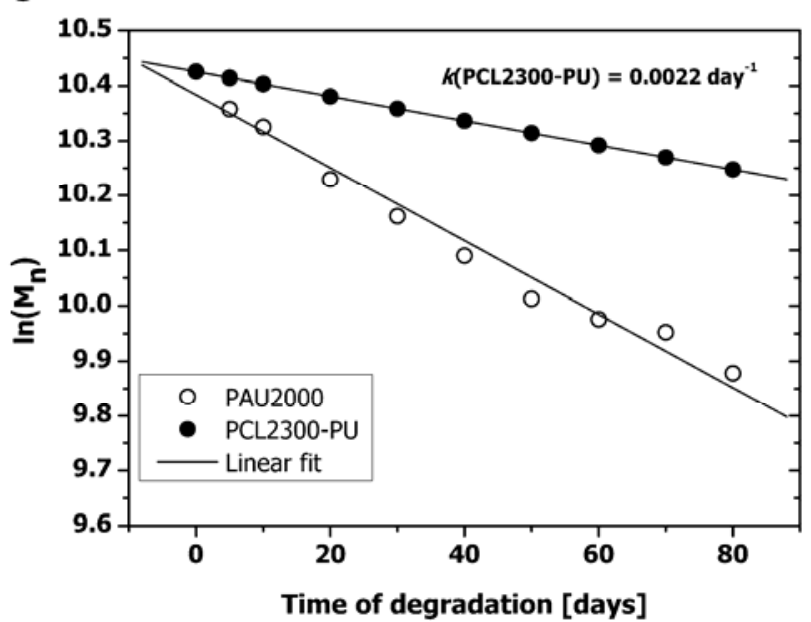


Figure 3. Hard segment hydrolysis.

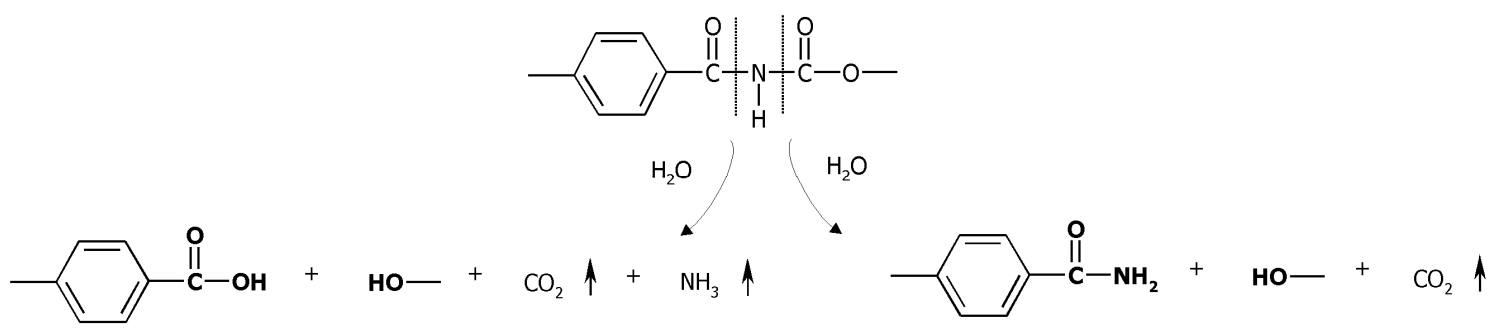

The observed deviation of the proposed pseudo-linear autocatalytic model and degradation of PAUs originates from the fact that the basic assumptions for this model were not fulfilled. The autocatalytic model was derived based on the following assumptions: (1) extent of chain scission is small, (2) only ester bonds hydrolyze and (3) there is no mass loss [19]. PAUs did loose mass upon degradation and in addition to the ester groups of the soft segment, acylurethane groups also might have hydrolyzed. Even though they were both composed of the same molar mass of the PCL soft segment, PAUs degraded much faster than PCL-PUs based on a BDI hard segment [18] (Figure 2). This can be explained by the chemical nature of the acylurethane moiety and the lower degree of microphase separation of PAUs compared to the PCL-PUs (see Section 2.3 for details).

The combination of mass loss and decrease of molar mass indicates that the hydrolytic degradation of PAUs occurs with combined bulk and surface erosion mechanisms.

\subsection{Thermal Properties of PAUs Upon Degradation}

Three PAUs with different PCL-oligodiol molar masses (PAU1000, PAU1500 and PAU2000) exhibited different melting endotherms (Figure 4).

Figure 4. Thermal properties of PAUs upon degradation at $37{ }^{\circ} \mathrm{C}$ in phosphate buffer solution ( $\mathrm{pH}=7.2)$. Heating endotherms (1st heating scan) of PAUs with different PCL-oligodiol lengths non-degraded ( 0 days) and at the end of the degradation period (80 days). $T_{g}$-glass transition temperature; $T_{m, S S}$-soft segment melting temperature; $T_{m, H S}$-hard segment melting temperature.

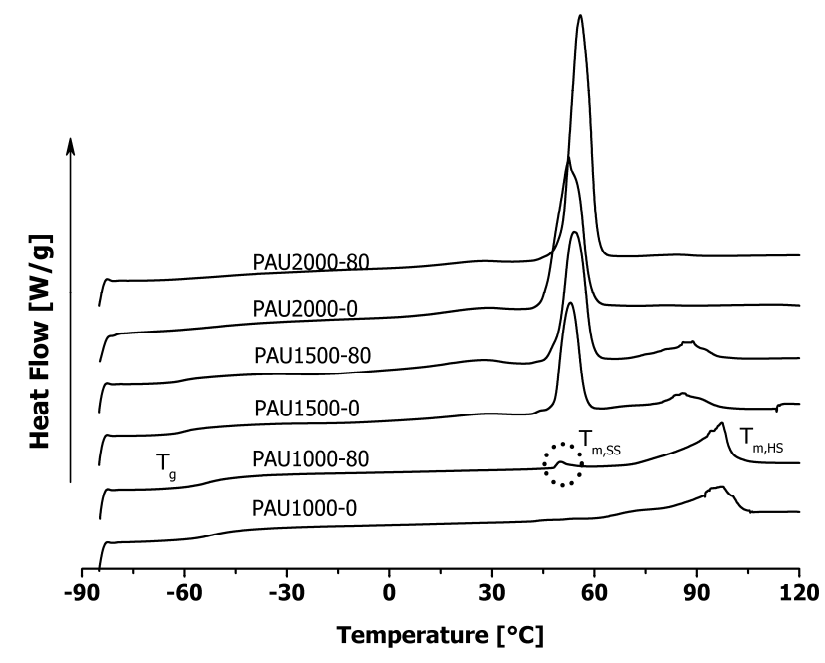


Analysis of the PAUs before the in vitro degradation revealed a microphase separated structure in the case of PAU1000 and PAU1500, characterized by a hard segment melting peak and the glass transition temperature $\left(T_{g}\right)$ of the soft segment (Figure 4). PAU2000 was not microphase separated, showing only a melting peak originating from crystalline PCL-soft segment, indicating that the preferred mechanical properties typical for polyurethanes were virtually absent.

Both PAU1500 and PAU2000 contained a relatively high fraction of crystalline PCL-soft segment (no $T_{g}$ detectable of the soft segment of PAU2000) which could retard the hydrolytic degradation both in vitro and in vivo. The appearance of the soft segment melting peak in PAU1000 from day 50 (Figure $4 \& 5$ ) on is a result of the chain scission and increased mobility of PCL-soft segments. Similar results have been observed earlier by different research groups $[1,2,20]$.

Figure 5. Soft segment and hard segment melt enthalpies of PAUs upon degradation. (a) Soft segment melt enthalpy $\left(\Delta \mathrm{H}_{\mathrm{m}, \mathrm{SS}}\right)$; (b) Hard segment melt enthalpy $\left(\Delta \mathrm{H}_{\mathrm{m}, \mathrm{HS}}\right)$.

A

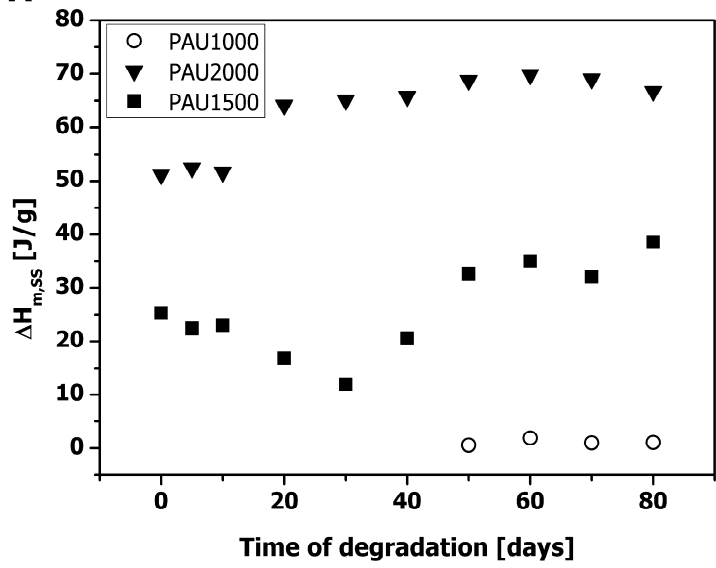

B

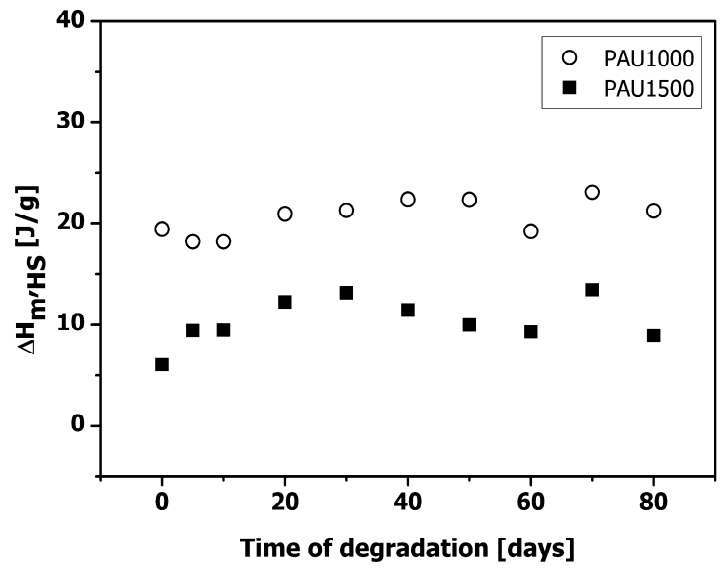

However, the amount of crystalline PCL even after 80 days of degradation was negligible (Figure 5a) and it was not expected to influence PAU1000 degradation to a great extent. The soft segment crystallinity of PAU2000 gradually increased with incubation, most probably because some chain scission had occurred in the amorphous phase of the PAU2000, allowing for higher chain mobility. In addition, the overall mobility of the chains might have been facilitated by the long incubation (annealing) in the humid environment at $37^{\circ} \mathrm{C}$.

PAU1000 exhibited the highest (bulk) hard segment crystallinity (Figure 5b), which remained constant during the course of degradation.

\subsection{Microphase Separation of PAUs Prior to Degradation (Trans-FTIR)}

Since hydrolytic degradation can only take place in the amorphous fraction of the polymer, the degree of microphase separation is one of the key factors that determine the hydrolysis rate of segmented polyester urethanes. To be able to determine which fraction of the hard segment was crystalline from the total urethane present (as a measure of the extent of the microphase separation), an enthalpy of melting of the $100 \%$ crystalline hard segment should be known. Since this was not the case, we attempted to calculate the extent of the microphase separation from the FTIR deconvolution analysis of the carbonyl absorption region of the PAUs. The carbonyl region was found to be suitable 
for this determination as described by Pretsch et al. [21]. Since only PAU1000 and PAU1500 exhibited microphase separation, PAU2000 was excluded from the FTIR analysis.

Bearing in mind that $\mathrm{H}$-bond associated $\mathrm{C}=\mathrm{O}$ always appears at the lower wavenumbers $[21,22]$, and correlating the deconvolution results with the DSC observations (Figure 5), overlapping peaks in the $\mathrm{C}=\mathrm{O}$ region were assigned as denoted in Figure 6. As already known from the literature, upon heating, dissociation of H-bonding occurs, followed by the disorder of the hard segment (HS) and soft and hard segment mixing $[23,24]$. The disruption of the HS crystallinity led to the decrease in the crystalline HS peak in both PAU1000 and PAU2000 (Figure 6). The decrease of the HS crystallinity was accompanied by the increase of the amorphous HS portion (Figure 6). As already observed by DSC, PAU1000 did not contain PCL soft segment (SS) in the crystalline form. The SS crystallinity of PAU1500 decreased upon heating, which resulted in the majority of the SS to be amorphous (Figure 6).

Figure 6. Carbonyl region of PAUs (transmission Fourier Transform Infra Red (FTIR)) prior to degradation. (a) Assignment of peaks of the PAU1000 carbonyl region curve fit; (b) PAU1000-integral values of peaks with temperature increase; (c) PAU15000-integral values of peaks with temperature increase.

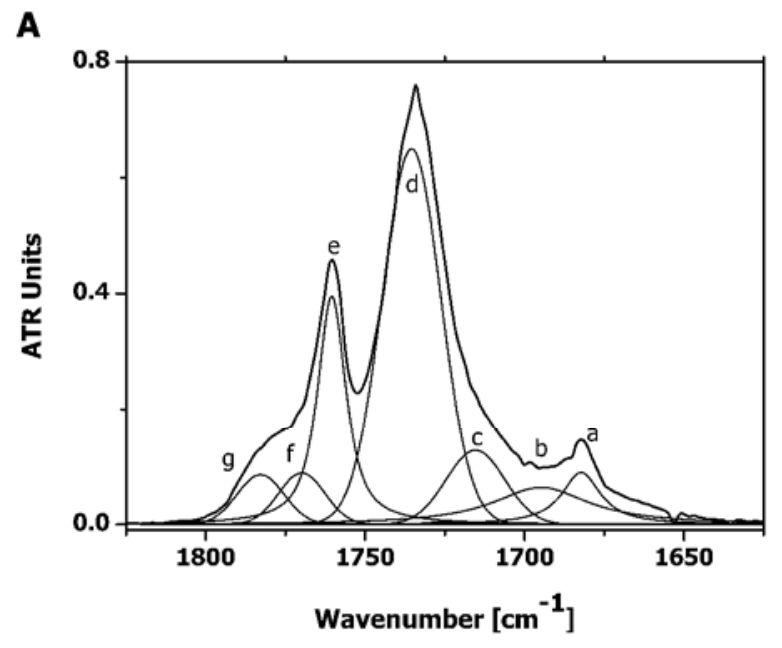

a - crystalline HS $\left(1683 \mathrm{~cm}^{-1}\right)$

b - amorphous HS $\left(1700-1718 \mathrm{~cm}^{-1}\right)$

c - crystalline SS $\left(1720-1725 \mathrm{~cm}^{-1}\right)$

d - amorphous SS (1730 - $\left.1735 \mathrm{~cm}^{-1}\right)$

e - symmetric acyl vib. HS $\left(1760 \mathrm{~cm}^{-1}\right)$

f - asymmetric acyl H-bonded HS $\left(1771 \mathrm{~cm}^{-1}\right)$

$\mathrm{g}$ - asymmetric acyl non. H-bonded HS $\left(1784 \mathrm{~cm}^{-1}\right)$

B

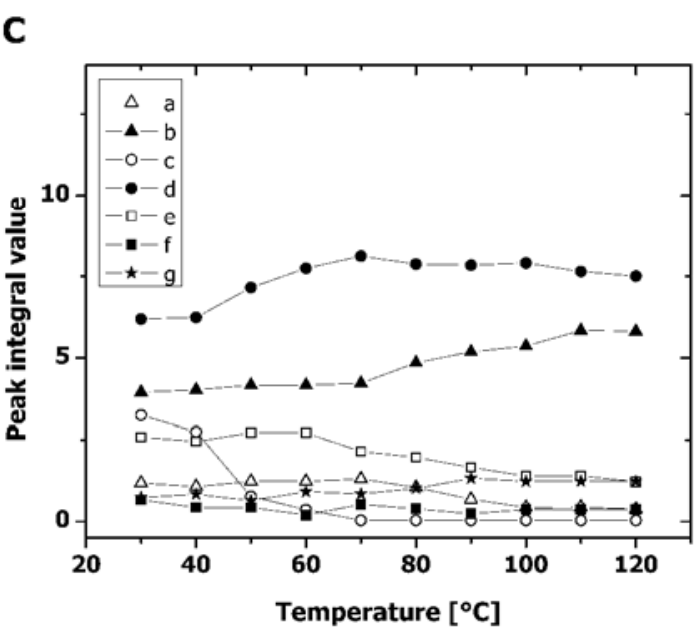

If we observe the molecular structure of the PAUs (Scheme 1), we could suspect that the acyl carbonyls and the phenyl ring could engage in a resonance interaction, which would further result in coplanar intermolecular orientation and four different conformations (two cis and two trans). Acyl 
carbonyls would than rotate relatively freely around the single bond and the differences in the levels of vibration energies specific for the different conformers would be small. However, if such a system gets fixed in any way, the difference in energy levels of the conformers would become larger, which would result in the splitting of the acyl carbonyl band. Sun et al. observed this phenomenon by analyzing chemically similar polymers, poly[di(butyl) vinylterephthalate] (PDBVT) [25]. Due to the attachment of the phenyl ring to a polyvinyl backbone, the energy levels became further apart, leading to acyl carbonyl band splitting [25].

The acyl carbonyl group of the PAUs is located next to the urethane bond, and participates in the hydrogen bonding (H-bonding) of the HS. Since H-bonding of the HS prefers certain conformations above other ones, rotation of the acyl carbonyls from both sides of the phenyl ring becomes restricted. Temperature increase results in the disturbance of the H-bonds of the HS in the crystalline phase, which is detected as a decrease of peaks representing acyl carbonyl in the crystalline form, and an increase of peaks originating from acyl carbonyl in the amorphous form. Since associated carbonyls always appear at lower frequencies than their non-associated counterpart [22], the peak at $1771 \mathrm{~cm}^{-1}$ has been assigned to H-bonded acyl carbonyl in the amorphous phase (Figure 6). The fact that these peaks increased with heating, indicated that it can only originate from the portion of the HS in the amorphous phase participating in the H-bonding with the amorphous SS. The other observed peak at $1784 \mathrm{~cm}^{-1}$ probably originates from the non-associated acyl carbonyl of the HS in the amorphous phase (Figure 6).

The HS crystallinity can be calculated using only peak integral values of the peaks originating from the urethane carbonyl group at $1680 \mathrm{~cm}^{-1}$ (Figure 6a, cryst. HS, peak designated as $a$ ) and $1718 \mathrm{~cm}^{-1}$ (Figure 6a, amorph. HS, peak designated as $c$ ). However, since the acyl carbonyl peaks are a part of the HS and participate in the HS H-bonding, it seemed more accurate to combine the effect of the two carbonyl groups. Therefore, the HS crystallinity can be calculated as:

$$
H S_{c y s t}=\frac{\int a+\int e}{\int a+\int b+\int e+\int f+\int g} \times 100 \%
$$

where $\int$ stands for integral values of the peaks $a, b, f, g$ and $h$ as designated in Figure 6a.

The percentage of the SS crystallinity can be calculated as given in the Equation 4.

$$
S S_{c r y s t}=\frac{\int d}{\int d+\int e} \times 100 \%
$$

As expected, a higher percentage of HS crystallinity was observed for PAU1000 with respect to PAU1500 (Table 1).

Table 1. Hard and soft segment crystallinity of non-incubated PAUs determined by trans-FTIR.

\begin{tabular}{lcc}
\hline Polymer & $\begin{array}{c}\text { HS cryst } \\
\text { (\%) }\end{array}$ & $\begin{array}{c}\text { SS cryst }^{* *} \\
(\mathbf{\%})\end{array}$ \\
\hline PAU1000-0 & 57.5 & 0.4 \\
PAU1500-0 & 41.3 & 34.4 \\
\hline
\end{tabular}

*Calculated according to Equation 3.

**Calculated according to Equation 4. 
In general, PAUs were less microphase separated than the PUs based on the same molar mass of PCL but with the HS comprised of BDI and BDO (HS cryst.(PCL1000-PU) $=73 \%$ and HS cryst.(PCL1600-PU) $=61 \%$ ) [26]. In addition to more hydrolysable HS, this lower degree of microphase separation renders PAUs more susceptible to hydrolysis.

\subsection{Microphase Separation of PAUs Upon Degradation (ATR-FTIR)}

Since PAU1000 was distinctly more microphase separated compared to the PAU1500, the morphology and the surface properties of PAU1000 were analyzed in more detail. ATR-FTIR, being a surface sensitive technique, provided information on the changes at the PAU1000 interface, which properties determine the protein deposition and the cell contact in vivo [14,15,27]. The change of surface properties upon degradation was followed by ATR-FTIR with utilization of the similar deconvolution method derived from the transmission measurements (Section 2.3). Due to optical complications, deconvolution of ATR-FTIR spectra can only be seen as a semi-quantitative technique. However, since we used this method to compare samples of the same polymer during degradation by identical manipulation of all the collected spectra, we consider the method to be acceptably credible. In order to emphasize the contribution of the acyl functionality to the hydrolysis of the PAUs, we also plotted the percentage HS crystallinity without the contribution of the acyl carbonyl peaks (denoted $e, f$ and $g$ in Figure 6a). This percentage was calculated according to the Equation 5.

$$
H S_{c y s t}=\frac{\int a}{\int a+\int c} \times 100 \%
$$

where $\int$ stands for integral values of the peaks $a$ and $c$ as designated in Figure 6a.

Compared to the HS crystallinity of non-degraded PAU1000 in the bulk (Table 1), crystallinity of the HS on the surface was lower (Figure 8) (in the bulk: $57.5 \%$, on the surface $\sim 30 \%$ ), which indicated that the polar, crystalline HS was initially located away from the surface. Although the overall crystalline SS content was low, it was predominantly present at the surface (in the bulk, Table 1: $0.4 \%$, on the surface, Figure 7: 5\%), which has been found before in other similar segmented polyurethanes [5,28].

With degradation, the crystallinity of both SS and HS increased at the surface. As already mentioned, hydrolysis can only occur in the amorphous portions of the PAUs. If the products of degradation would not diffuse out, the crystallinity of both SS and HS would remain the same. However, we did observe mass loss (Figure 1), which can solely be ascribed to the hydrolysis of the amorphous SS and HS and the diffusion of the degradation products preferentially from the surface. The SS crystallinity at the surface increased for $\sim 30 \%$ after 80 days of degradation (Figure 7 ), which was far more than observed in the bulk (Figure 5a). As already mentioned, PAUs degraded partially via surface erosion mechanism. Preferential hydrolytic chain scission at the surface probably induced higher chain mobility at the surface that allowed for SS crystallization. PCL crystallization upon degradation was also observed by Lam et al. [2] and Antheunis et al. [20]

Similar to the case of the SS crystallinity increase of PAU1000, the higher chain mobility at the surface of PAU1000 might also have allowed for better HS alignment, resulting in higher HS crystallinity at the surface relative to the bulk. Therefore, the increased crystallinity of both SS and HS upon degradation was most probably the result of both effects: Removal of the degradation products 
(mass loss) formed by ester and urethane bond hydrolysis in the amorphous portion of the PAUs, and the effect of the re-crystallization due to the higher mobility at the surface. The increased hydrogen-bonding capable hard HS content is expected to render the surface more hydrophilic, which is shown to further enhance cell adhesion [29,30].

Figure 7. Percentage hard (cryst. HS) and soft segment crystallinity (cryst. SS) upon degradation of PAU1000. ( $\circ$ ) Soft segment (SS) crystallinity (calculated according to Equation 4); $(\Delta)$ hard segment crystallinity with the contribution of the acyl carbonyl peak (HS-with Acyl) (calculated according to Equation 3); ( $\boldsymbol{\Delta}$ ) hard segment crystallinity without contribution of the acyl carbonyl peak (HS-without Acyl) (calculated according to Equation 5).

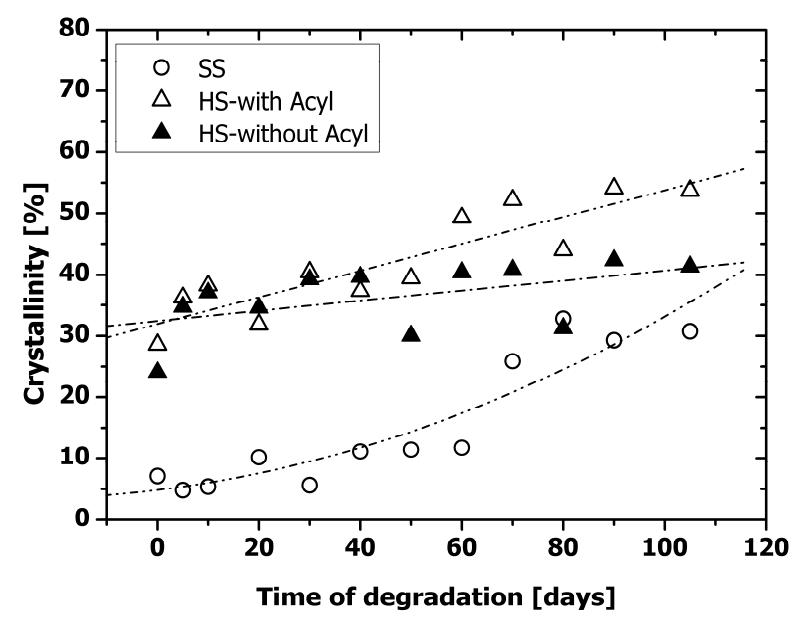

\subsection{Contact angle Measurements}

The increase in overall crystallinity at the surface of PAU1000 upon in vitro degradation and surface erosion were expected to result in the increased surface roughness. An increase of surface roughness can be detected as the contact angle increase. However, hydrolysis created polar functional groups (carboxyl, hydroxyl or amide) and increased HS content at the surface of the degraded PAU1000, both contributing to an increased hydrophylicity, which was observed by a decrease of the contact angle (Figure 8a).

Somewhat lower contact angle values for non-incubated sample most probably originated from the different sample treatment in comparison to the rest of the data collected in the course of degradation (Figure 8a). After 50-60 days of degradation, the contact angle of PAU1000 was $\sim 65^{\circ}$, which was close to optimal hydrophilicity conditions for cell adhesion and proliferation [29,30].

To model the biological environment of the potential PAU implant in vivo, the changes of the surface properties of PAU1000 in contact with biological fluids was investigated (Figure 8b). Coated on the Thermanox ${ }^{\circledR}$ cover slip, the contact angle of the PAU1000 in a dry state was $78.0^{\circ} \pm 2.6^{\circ}$ while the angle of the Thermanox ${ }^{\circledR}$ cover slips was $71.1^{\circ} \pm 1.6^{\circ}$ (Figure $8 \mathrm{~b}$ ). After incubation in PBS at $37^{\circ} \mathrm{C}$ for $1 \mathrm{~h}$, and $18 \mathrm{~h}$ the contact angle had slightly decreased, but after the incubation in ECM and FCS a noticeable increase of surface hydrophylicity, compared to the non-incubated sample, was observed, with the contact angle of PAU1000 incubated in FCS reaching $45.0^{\circ} \pm 7.2^{\circ}$ (Figure $8 \mathrm{~b}$ ). This 
increase of PAU1000 hydrophylicity could explain the best HUVEC adhesion result at longer culture time. After $18 \mathrm{~h}$ incubation in ECM, the PAU1000 surface contact angle was $60^{\circ}$, which is, according to the literature, an optimal value for the best cell adhesion [29]. Most probably surface hydrophobicity changed due to an increase in hard segment content on the PAU1000 surface as a result of the interaction of hard segment with water and proteins at the interface in combination with products of the surface erosion process.

Figure 8. (a) Contact angle of PAU1000 during in vitro degradation; $n=5$; data expressed as mean \pm standard error of the mean; (b) Contact angle of PAU1000 exposed to biological fluids: Phosphate buffer saline (PAU1000 in PBS), endothelial cell culture medium (PAU1000 in ECM) and fetal calf serum (PAU1000 in FCS). References included: non-incubated PAU1000 (Non-incub. PAU1000) and non-incubated Thermanox ${ }^{\circledR}$ (Non-incub. Therm). All the values, except where indicated (n.s.) were statistically significantly different $(n=5$; data expressed as mean \pm standard error of the mean).

A

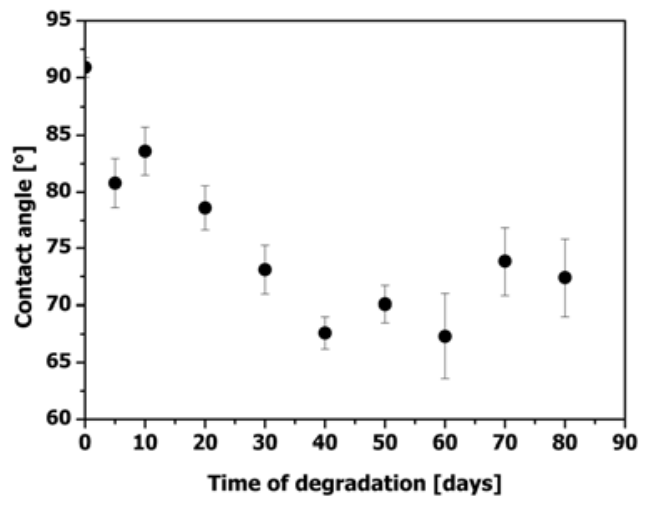

B

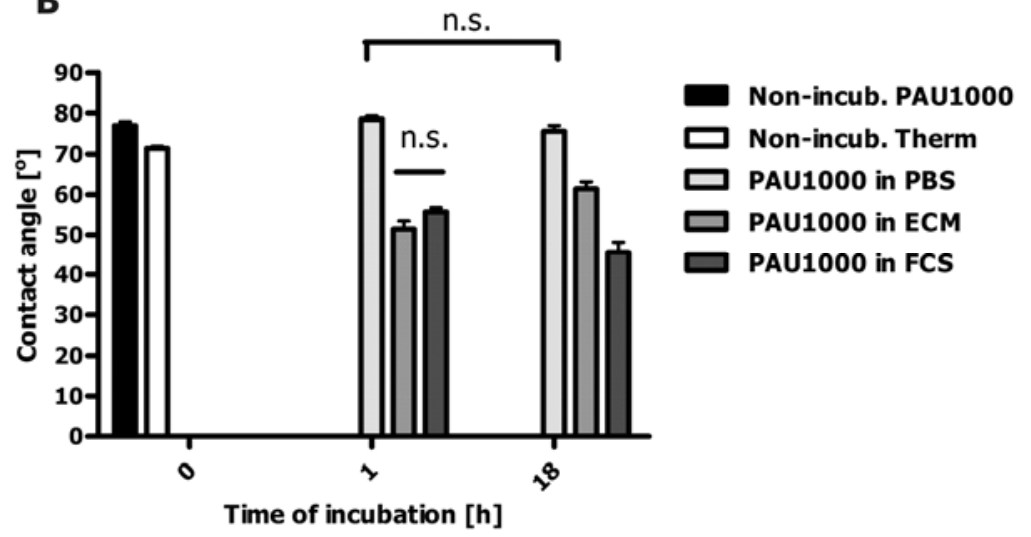

Upon implantation, PAU1000 performance is expected to be a result of a complex interplay between the material surface properties, protein deposition and a type of cells involved.

\subsection{Cytotoxicity (MTS Assay)}

In this study, we performed a reliable and robust method of determining cytotoxicity of the novel polyacylurethane PAU1000. Mitochondrial activity of PK-84 cells cultured in the medium containing extracts from PAU1000 was similar to both positive control and the blank, which was set to $100 \%$, i.e., non-toxic, showing that PAU1000 was non-cytotoxic (Table 2).

Table 2. Cytotoxicity determination. Cytotoxicity of PAU1000 was comparable to clinical grade polyurethane Pellethane ${ }^{\circledR}$. Controls were Latex (cytotoxic) and medium (non-toxic) $(\mathrm{n}=4$, data expressed as average $\pm \mathrm{SD})$.

\begin{tabular}{lc}
\hline [Test material & Cell survival [\%] \\
\hline PAU1000 & $86 \pm 6$ \\
Pellethane ${ }^{\circledR}$ & $91 \pm 6$ \\
Latex & $38 \pm 1$ \\
Medium & $104 \pm 13$ \\
\hline
\end{tabular}




\subsection{Haemocompatibility}

The rate of thrombin generation by PAU1000 was similar to low density polyethylene, which is lowly thrombogenic, and much lower than polydimethylsiloxane, which served as i.e., thrombogenic control material (Figure 9a). The other PCL-based PU, PU1000, was the least thrombogenic of all the materials tested. Since both the clotting cascade and platelet phospholipids were involved in this test, the results are clinically relevant. The good haemocompatibility renders PAU1000 a promising candidate for cardiovascular applications, e.g., bioartificial vascular grafts.

Complement convertases are formed by incubating biological fluids, blood or blood products with biomaterials, allowing the activation of the complement system and binding of complement convertases to the biomaterial surface. Surface activity of the C5 convertase indicates generation of C5a fragments, which are strong anaphylactic and chemotactic components and initiation of C5b-9, the terminal complement complex with cytotoxic capacity. Activation of the complement cascade in the presence of PAU1000 was measured through the activity of complement C5 convertase on the material surface. In this respect, PAU1000 only minimally activated complement compared to the other control biomedical materials (Figure 9b). Both PUs induced very low complement convertase activity. Therefore, the low C5 convertase activity recorded in the experiment with PAU1000 would be predicted to induce only a minimal inflammatory reaction in vivo.

Figure 9. Haemocompatibility assessment. (a) Thrombin generation assay; (b) C5 complement convertase activity. Control materials used for comparison: 1,4-butanediisocyanate based polyurethane (PU1000), polydimethylsiloxane (PDMS) and low density polyethylene (LDPE) as the Gold standard (mean values of duplicate measurements $\pm \mathrm{SD}$ ).
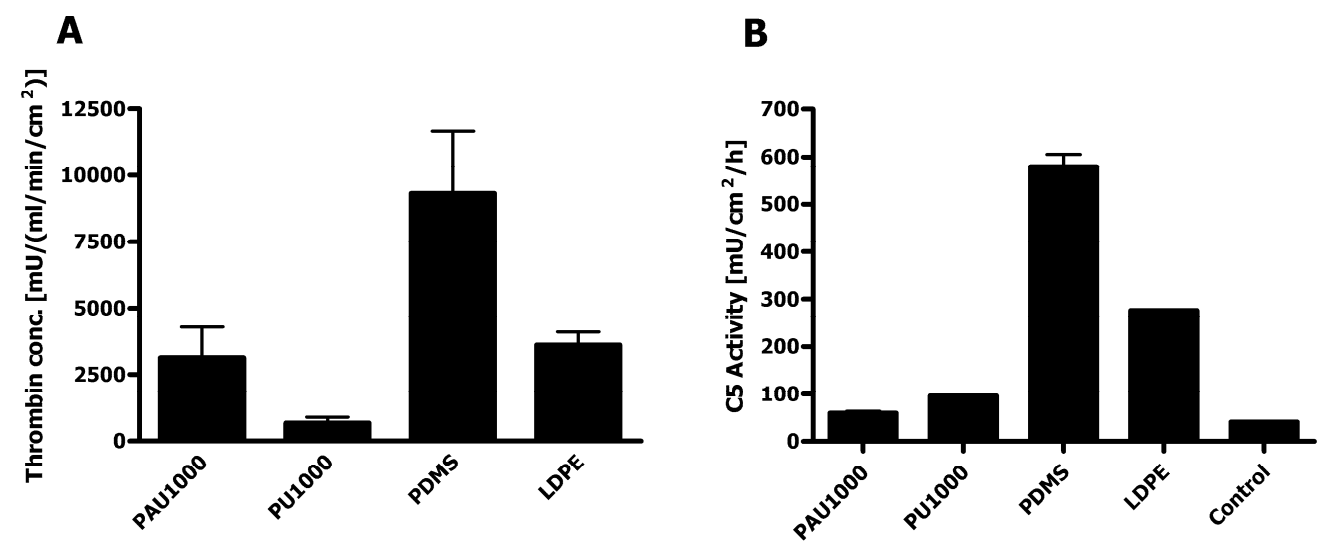

\subsection{HUVEC Adhesion to PAU1000}

Adhesion of human umbilical vein endothelial cells (HUVEC) to PAU1000 was monitored for $18 \mathrm{~h}$. Based on the hydrophobic nature of PAU1000, we expected only a low adhesion of HUVEC to the PU. However, the difference in contact angle values between PAU1000 $\left(78.0^{\circ} \pm 2.6^{\circ}\right)$ and Thermanox $^{\circledR}$ cell culture treated cover slips $\left(71.1^{\circ} \pm 1.6^{\circ}\right)$ was similar. Thus, in this setting hydrophobicity was not the only parameter governing the cell adhesion. We assume that the presence of carboxyl functionality on the surface of Thermanox ${ }^{\circledR}$ cover slips rendered the surface more cell-adhesive. In order to further enhance cell adhesion, we included samples that were additionally coated with fibronectin. Fibronectin 
possesses integrin binding motifs such as the RGD (Arg-Gly-Asp) sequence that could augment the attachment of HUVEC [31,32]. Initially, the number of adhered cells increased regularly with time, irrespectively of Fn-coating, with Fn-coated PAU1000 cover slips providing faster cell adhesion for the period up to $4 \mathrm{~h}$ (Figure 10a).

Figure 10. Adhesion and proliferation of endothelial cells (HUVEC) on PAU1000. (A) HUVEC adhesion to PAU1000 (a) and Fn-coated PAU1000 ( $\boldsymbol{\Delta})$ monitored up to 18 h. ( $\mathrm{n}=3 ; 6$ images per cover slip, data expressed as mean \pm standard error of the mean; asterisks indicate significant differences $* \mathrm{p}<0.05$ ); (B) Viability test of HUVEC during adhesion to PAU1000 and Fn-coated PAU1000 (PAU1000+Fn) at 4 and $8 \mathrm{~h}(\mathrm{n}=2$; 6 images per cover slip, data expressed as mean \pm standard error of mean; asterisks indicates significant difference * ${ }^{*}<0.05$ ); (C) HUVEC proliferation after $25 \mathrm{~h}$ culture on PAU1000, Fn-coated PAU1000, Thermanox ${ }^{\circledR}$ cover slips (Therm) and Fn-coated Thermanox ${ }^{\circledR}$ cover slips (Therm+Fn) $(n=2 ; 5$ images per cover slip, data expressed as mean \pm standard error of the mean).
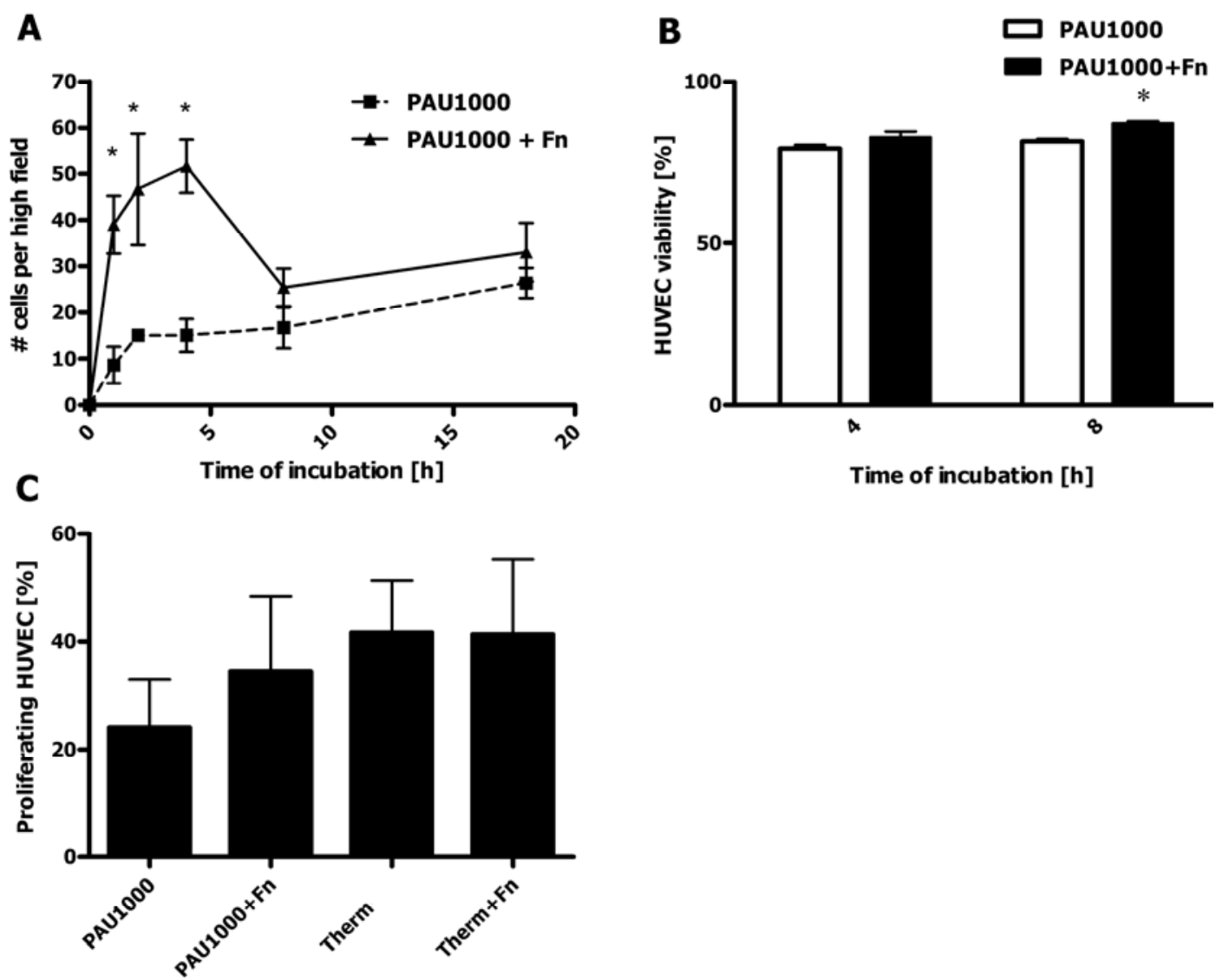

HUVEC cultured on PAU1000 exhibited a steady increase in the number of adhered cells, resulting in a similar degree of adhered HUVEC on both Fn-coated and bare PAU1000. In order to elucidate the observed phenomenon of decrease of cell adhesion between the points $4 \mathrm{~h}$ and $8 \mathrm{~h}$, the viability was measured using Trypan blue dye exclusion. The number of viable cells that adhered after incubation was at both time points more than $80 \%$ (Figure 10b), which was similar to the other time points (data not shown). Although the cells remained viable throughout the test period, some had detached from the Fn-coated slips after $8 \mathrm{~h}$ of culture. Since Fn is hydrophobic, a property that does not depend on the 
nature of the underlying substrate [33], the hydrophobic interaction of Fn with PAU1000 is strong. These strong hydrophobic interactions could have caused adverse conformational changes in the Fn molecules that impaired cell adhesion. Furthermore, the Fn-coating might have been partially lost during prolonged culturing, thus exposing the cells to the less adhesive bare surface of the PAU1000. In the later stages of incubation, adhering HUVEC started producing their own adhesive extracellular matrix molecules such as collagen and fibronectin (data not shown) [34]. This cellular activity might be the reason why with prolonged culture time (overnight), the difference between the HUVEC adhesion to bare PAU1000 and Fn-coated PAU1000 was diminished (Figure 10a).

A possible explanation for low cell adhesion to PUs could be that the PU surface exposed to plasma proteins is mostly covered with proteins that are generally considered non-adhesive. Fibrinogen is initially adhered, but it is competed for deposition on the polyurethane surface with albumin, high-density lipoprotein and high molecular weight kininogens (HMWK), which all do not favor cell binding [35]. The dynamics of the rearrangement and replacement of proteins on the PU surface is determined by the hydrophobicity and the degree of polyurethane micro-phase separation [10,34,36,37]. This influences protein mobility and rearrangement for better recognition by cell integrins [34]. Proteins interact with the polyurethanes via hydrophobic interactions (soft segment domains) and/or hydrogen bonding (hard segment). Highly hydrophobic substrates bind more protein as compared to more hydrophilic substrates. The hard segments are reported to bind fibrinogen via hydrogen bonds [36]. Being more adhesive, the hard segment is also known to interact with platelets and lead to their activation [28,37]. To contribute to the complexity of polyurethane-protein and cell interaction, a polyurethane surface is also dynamic and might rearrange to promote better adhesive properties by exposure of hard segments. A dry PU surface, being in contact with air, which is hydrophobic, has soft segments mostly exposed [5]. Upon immersion in water, more polar hard segments capable of hydrogen bonding migrate to the surface, making it more hydrophilic. The decrease in contact angle was observed for PAU1000 upon contact with biological fluids (Figure 8).

\subsection{HUVEC Proliferation on PAUS}

Even though the rate of HUVEC adhesion may appear low, longer incubation experiments of $25 \mathrm{~h}$ revealed that both bare PAU1000 and Fn-coated PAU1000 allowed for significant HUVEC proliferation, reaching almost $50 \%$ cellular confluence already after $25 \mathrm{~h}$ of culture, which was similar to the Thermanox ${ }^{\circledR}$ cover slips (Figure 10c). That indicates that fast and efficient in vitro endothelialization of grafts fabricated from PAU1000 is feasible. The additional coating with Fn appeared to further increase the number of proliferating cells on PAU1000, although it had little influence on Thermanox ${ }^{\circledR}$ cover slips.

\section{Experimental Section}

\subsection{Materials}

$\varepsilon$-Caprolactone (CL) was obtained from Union Carbide (Terneuzen, The Netherlands) and was purified by distillation under reduced pressure from calcium hydride $\left(\mathrm{CaH}_{2}\right)$. Terephthaloyl diisocyanate was synthesized from terephthalamide, obtained from TCI (Japan) and oxalyl chloride 
from Sigma-Aldrich (Zwijndrecht, The Netherlands) using a modified method of Tsuge et al. [38]. Analytical grade 1,4-butanediol (BDO) was purchased from Sigma-Aldrich (Zwijndrecht, The Netherlands) and purified by distillation from $3 \AA$ mole sieves under reduced pressure. Chloroform, diethyl ether, dimethyl formamide and n-hexane as analytical grade solvents were purchased from Acros Organics (Geel, Belgium) and were used without further purification. Sodium azide was purchased from Sigma-Aldrich (Zwijndrecht, The Netherlands) and used as received.

Polyacylurethane films were cast from chloroform onto PFA Petri dishes (perfluoroalkoxy polymer resin) obtained from Bergof (Florida, USA).

Thermanox ${ }^{\circledR}$ cover slips which were purchased from NUNC ${ }^{\mathrm{TM}}$ (Roskilde, Denmark).

Glutaraldehyde was purchased from Sigma-Aldrich (Zwijndrecht, The Netherlands). RPMI medium and fetal calf serum (FCS) were obtained from Cambrex Bio Science (Verviers, Belgium). CellTiter $96^{\circledR}$ Aqueous One Solution A was purchased from Promega Corporation (Madison, WI, USA). Polyurethane Pellethane ${ }^{\circledR}$ was a gift from Dow Chemicals (Midland, MI, USA).

The following materials were used in the haemocompatibility study: Polydimethylsiloxane (PDMS) from Eriks (Alkmaar, The Netherlands), low-density polyethylene (LDPE) ET311350 from Goodfellow (Cambridge, UK), thrombin chromogenic substrate $\mathrm{S}_{2238}$ and chromogenic substrate $\mathrm{S}_{2527}$ from Chromogenix (Milano, Italy).

In cell adhesion and proliferation tests the following materials have been employed: Diamidino-2-phenylindol-dihydrochlorid (DAPI) from Sigma-Aldrich (Germany), Triton X-100 from Sigma (St. Louis, USA), polyclonal antibody rabbit-anti Ki-67 from Nova Castra Laboratory (Newcastle, UK), Tween 20 from Sigma-Aldrich (Zwijndrecht, The Netherlands), Avidin/Biotin blocking kit from Vector Laboratories Inc. (CA, USA), streptavidin-FITC from DAKO (The Netherlands) and Citifluor API from Agar Scientific (Essex, UK).

\subsection{Synthesis of Polyacylurethanes}

Polyacylurethanes (PAUs) were synthesized by a slightly modified method of Heijkants et al. [8]. The synthesis was performed in two steps: 1) oligodiol synthesis and 2) polymerization of the oligodiol with terephthaloyl diisocyanate (TPHDI) (Scheme 1). The oligodiols, poly( $\varepsilon$-caprolactone) (PCL) diols of three different lengths $(1000,1500$ and $2000 \mathrm{~g} / \mathrm{mol})$ were prepared by thermal polymerization employing 1,4-butanediol as an initiator without the use of any catalyst at $150{ }^{\circ} \mathrm{C}$ for 7 days under argon [26]. The second step was performed in the Micro Twin Extruder. Briefly, a powder mixture of PCL (molar mass $=1000 \mathrm{~g} / \mathrm{mol}$ ) (approximately $7.0 \mathrm{~g} ; 7.0 \mathrm{mmol}$ ) and TPHDI (0.75 g; $3.5 \mathrm{mmol}$ ) was fed to a micro-extruder at $65{ }^{\circ} \mathrm{C}$. Subsequently, the extruder was heated up to $130{ }^{\circ} \mathrm{C}$ and another portion of TPHDI $(0.75 \mathrm{~g})$ was added. The average polymerization time was 6 minutes; the resulting polymer was collected, purified by precipitation in diethyl ether from chloroform solution, and dried in a vacuum stove at $40{ }^{\circ} \mathrm{C}$. The reaction scheme is depicted in Scheme 1. 
Scheme 1. Synthesis of polyacylurethanes. Terephthaloyl diisocyanate (TPHDI) and poly( $\varepsilon$-caprolactone) (PCL) are reacted without a catalyst to yield polyacylurethane (PAU).

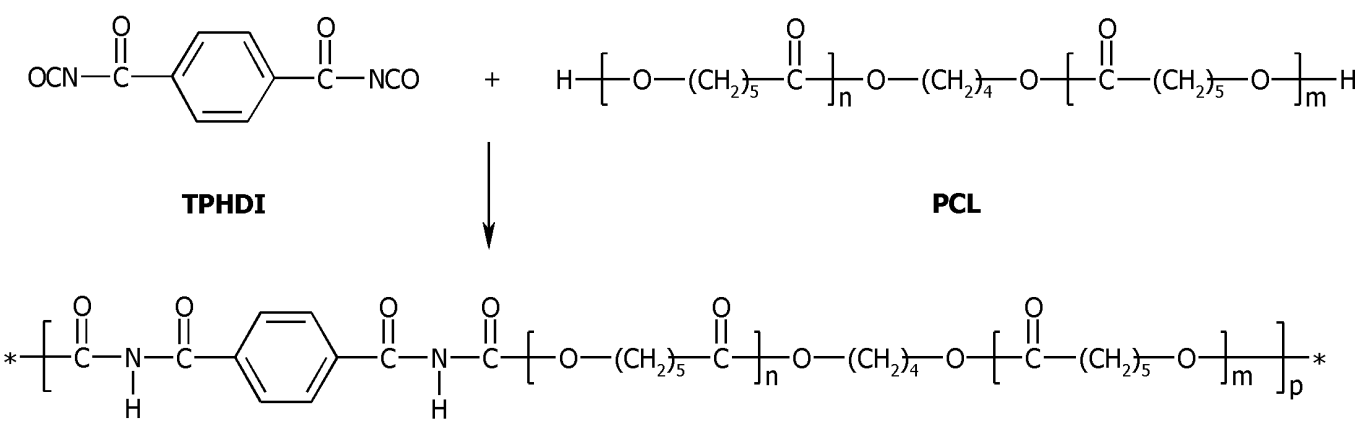

Polyacylurethane (PAU)

\subsection{Preparation of Polymer Films}

Polymer films were prepared by casting a $2 \mathrm{w} / \mathrm{v} \%$ polyurethane solution in chloroform onto either ID $8 \mathrm{~cm}$ PFA Petri dishes or Thermanox ${ }^{\circledR}$ cover slips (treated side up) at room temperature. Both the freestanding films and the coated slips were washed in a Soxhlet apparatus utilizing n-hexane as the extraction solvent for $16 \mathrm{~h}$ in order to remove any surface contaminants such as potentially present silicones. Finally, the materials were dried and stored in the vacuum stove at $40{ }^{\circ} \mathrm{C}$ until further use.

In some of the experiments, PAU-coated Thermanox ${ }^{\circledR}$ cover slips were additionally coated with fibronectin (Fn). In brief, $250 \mu \mathrm{L}$ of 1 weight\% solution of recombinant human Fn in PBS was placed in wells (24-wells culture plate) containing PAU-coated slips and incubated at $37{ }^{\circ} \mathrm{C}$ for $30 \mathrm{~min}$. The excess of Fn-solution was aspirated and the adhered fibronectin was cross-linked with $0.5 \%$ glutaraldehyde (GA) solution in PBS at room temperature for $15 \mathrm{~min}$. In order to remove all the GA remains, all the coated samples were extensively washed with PBS and endothelial cell culture medium (ECM medium) formulated in our laboratory. The endothelial culture medium (ECM) consisted of RPMI 1640 supplemented with $20 \%$ heat-inactivated FSC, $2 \mathrm{mM}$ L-glutamine, $5 \mathrm{U} / \mathrm{mL}$ heparin, $100 \mathrm{IE} / \mathrm{mL}$ penicillin and $100 \mu \mathrm{g} / \mathrm{mL}$ endothelial growth factor supplement extracted from bovine brain.

\subsection{In Vitro Degradation Set-Up}

Films obtained as described above were cut in quarters and weighed. Subsequently, each piece was placed in a glass container and $100 \mathrm{~mL}$ of phosphate buffer (solution in water, $\mathrm{pH}=7.2$; Sigma-Aldrich, Zwijndrecht, The Netherlands) solution, containing $0.02 \mathrm{wt} \%$ of sodium azide, was added. Labeled and well-closed containers were placed in a dark water bath at $37{ }^{\circ} \mathrm{C}$. At predetermined time points, starting from 5 up to 80 days, samples ( $\mathrm{n}=2$ per time point) were taken and rinsed with distilled water and blotted gently with tissue paper to remove surface water. Subsequently, the samples were dried in a vacuum oven at $40{ }^{\circ} \mathrm{C}$. The weight was monitored until it reached a constant value. 


\subsection{Mass Loss}

The mass loss of PAU-films upon in vitro degradation was determined by weighing the samples (one quarter of the film) at predestined time points and calculated as follows:

$$
\text { Mass loss }=\frac{\left(m_{0}-m_{d}\right)}{m_{0}} \times 100 \%
$$

Where $m_{o}$ is the dry mass before incubation and $m_{d}$ is the dry mass after degradation.

\subsection{Molar Mass}

The molar masses of the PAUs after synthesis and purification, as well as the molar masses of samples ( $\mathrm{n}=2$ films per time point) upon degradation were determined by gel permeation chromatography (GPC) utilizing a Waters 600 Powerline system, equipped with 2 mixed-C Pl-gel $5 \mu$ columns employing dimethyl formamide (DMF) with $0.01 \mathrm{M} \mathrm{LiBr}$ as eluent at $70{ }^{\circ} \mathrm{C}$. The data analysis was done using conventional calibration with polystyrene standards accompanied by in-house software. Number average molar mass $\left(M_{n}\right)$ data (average of two samples per time point) were fitted by using the OriginPro 7.5 software.

\subsection{Thermal Properties of PAUs}

Thermal properties of PAUs as polymerized and at different time points of degradation were measured with a differential scanning calorimeter Q 1000 from TA Instruments. The samples with masses varying between $7-10 \mathrm{mg}$ were heated from $-85^{\circ} \mathrm{C}$ to $+150{ }^{\circ} \mathrm{C}$ with a rate of $10{ }^{\circ} \mathrm{C} / \mathrm{min}$. The data collected during the first heating run were analyzed utilizing the TA Instruments software version 4.0.

\subsection{Fourier-Transform Infra Red Spectroscopy (FTIR)}

All the infrared spectral manipulation was performed using the Opus v.4.2 software package (Bruker Optik GmbH).

Infrared transmission measurements were done on films cast on $\mathrm{KBr}$ pellets from chloroform solution using a Bruker IFS88 spectrometer equipped with a MCT-A detector at the resolution of $2 \mathrm{~cm}^{-1}$. The $\mathrm{KBr}$ pellets were measured horizontally using the Bruker infrared microscope accessory. Five hundred scans were recorded per spectrum. Temperature was varied from room temperature to $120^{\circ} \mathrm{C}$.

The carbonyl absorption region from $1850 \mathrm{~cm}^{-1}$ to $1575 \mathrm{~cm}^{-1}$ was deconvoluted by fixing the peak position and allowing for peak intensity, width and shape to be optimized by the Opus software. The Levenberg-Marquardt algorithm was used in curve fit optimization. The calculated residual RSM fitting error was always $<0.009$.

ATR-FTIR was done using a Bruker IFS88 spectrometer equipped with a Golden Gate (Graseby Specac) single reflection ATR accessory. Spectral resolution was $4 \mathrm{~cm}^{-1}$ and 50 scans were taken per spectrum. 


\subsection{Contact angle Measurements}

Surface properties of PAU1000 were assessed during the in vitro degradation up to a period of 80 days and upon short-term exposure to biological fluids. For the latter, PAU1000-coated Thermanox ${ }^{\circledR}$ cover slips were placed in the wells of a 24 -well culture plate and incubated in PBS, ECM supplemented with $20 \% \mathrm{FCS}$ and $100 \% \mathrm{FCS}$ at $37{ }^{\circ} \mathrm{C}$ for $1 \mathrm{~h}$, and $18 \mathrm{~h}$. In both cases, after incubation the samples were rinsed by means of spraying distilled water against both sides of the samples. The samples were blotted with tissue paper, free-standing film or cover slips were fixed to glass slides by double-sided adhesive tape and contact angle was measured using a sessile drop method using a Krüss Drop Shape Analysis System DSA 10.

\subsection{MTS Cytotoxicity Assay}

To perform cytotoxicity assays, a fibroblast cell line PK84 was cultured in RPMI medium containing 10\% FCS. Cells seeded in a density of 5,000 cells/well were exposed to an extract of PAU1000, obtained by shaking the material overnight in culture medium at $37^{\circ} \mathrm{C}$. After $48 \mathrm{~h} 20 \mu \mathrm{L}$ of CellTiter $96^{\circledR}$ Aqueous One Solution A was added to each well and the absorbance intensity was recorded at $490 \mathrm{~nm}$ after 90 min of culture. Pellethane ${ }^{\circledR}$ and latex were used as negative and positive control, respectively.

\subsection{Haemocompatibility of $P A U$}

Two different methods were employed to assess the blood compatibility of PAU1000: Thrombin generation assay and Complement convertase activity. Another biomedical polyurethane developed in our laboratory by Heijkants et al. [26] (1,4-butanediisocyanate-based (BDI) hard segment and PCL-based soft segment) with the same PCL length as PAU1000, and commonly used reference materials were included in the experiments.

The formation of thrombin in the presence of the biomaterial was determined by means of the Thrombin Generation Assay (TGA) as developed by Haemoscan (Groningen, The Netherlands). Polydimethylsiloxane (PDMS) and low-density polyethylene (LDPE) were used as positive and negative control, respectively. Thrombin generation was obtained in citrate plasma depleted of fibrinogen. Materials (surface area $0.5 \mathrm{~cm}^{2}$ ) were incubated in duplicate in $350 \mu \mathrm{L}$ plasma in polyethylene tubes for $15 \mathrm{~min}$ at $37^{\circ} \mathrm{C}$. Then $\mathrm{CaCl}_{2}(30 \mathrm{mM})$ and phospholipids were added, gently mixed and after 1, 2, 4 and $6 \mathrm{~min}, 10 \mu \mathrm{L}$ of the incubation mixture was diluted in $490 \mu \mathrm{L}$ ice cold $25 \mathrm{mM}$ Tris- $\mathrm{HCl}$ buffer to stop further thrombin formation or inhibition. These diluted samples were incubated at $37^{\circ} \mathrm{C}$ with $3 \mathrm{mM}$ thrombin chromogenic substrate $\mathrm{S}_{2238}$ for $20 \mathrm{~min}$. The optical density of the yellow color was measured at $405 \mathrm{~nm}$ in a micro titer plate reader from Powerwave 200 Bio-Tech Instruments (Winooski, Vermont). A calibration curve was made with known concentrations of thrombin in Tris buffer.

Surface-bound C5 convertase was determined on $1 \mathrm{~cm}^{2}$ material after incubation in porcine plasma for $15 \mathrm{~min}$ at room temperature (CCA, Haemoscan, Groningen, The Netherlands). After incubation, the samples were rinsed and incubated in chromogenic substrate $\mathrm{S}_{2527}$ ) diluted in TRIS buffer at room 
temperature in the dark for $24 \mathrm{~h}$. Thereafter, the optical density was determined at $405 \mathrm{~nm}$ in a microplate reader.

\subsection{HUVEC Adhesion and Viability on PAU1000}

Human umbilical vein endothelial cells (HUVEC) were isolated and cultured as previously described [39]. In short, the cells were cultured on endothelial culture medium (ECM; see Section 3.3) and $1 \%$ gelatin coating at $37{ }^{\circ} \mathrm{C}$ and $5 \% \mathrm{CO}_{2}$. Polyurethane (PAU1000)-coated Thermanox ${ }^{\circledR}$ cover slips and additionally fibronectin (Fn)-coated cover slips were placed in 24-well plates and HUVEC $\left(130.000 / \mathrm{cm}^{2}\right)$ were seeded in each well. After $18 \mathrm{~h}$ of culture, adhered cells were fixed in $2 \%$ paraformaldehyde (PFA) in PBS at room temperature for $20 \mathrm{~min}$. Cells were stained with DAPI in PBS for $30 \mathrm{~min}$. The cell number was determined by fluorescence microscopy utilizing Leica DC 300F apparatus (Wetzlar, Germany). For the viability test, HUVEC $\left(150.000 / \mathrm{cm}^{2}\right)$ in ECM were seeded onto either PAU1000-coated cover slips, or Fn-coated PAU1000 cover slips, and cultured for 4 and 8 h. The adhered cells were detached with trypsin and the viability was assessed using Trypan blue staining.

\subsection{HUVEC Proliferation on PAU1000}

The HUVEC proliferation was evaluated upon culture on PAU1000, Fn-coated PAU1000, bare Thermanox $^{\circledR}$ cover slips and Fn-coated Thermanox ${ }^{\circledR}$ cover slips for $25 \mathrm{~h}$. After culture, the non-adhered cells were removed by washing and the attached cells were fixed with $2 \%$ PFA in PBS and stored dry at $4{ }^{\circ} \mathrm{C}$. For the staining, samples were thawed, dried and additionally fixed with $2 \%$ PFA in PBS. Fixed cells were permeabilized with $0.5 \%$ Triton X-100 in PBS. Samples were then incubated with polyclonal antibody rabbit-anti Ki-67 (1:500) at room temperature for $90 \mathrm{~min}$. Subsequently, cells were washed with $0.05 \%$ Tween 20 in PBS. Endogenous avidin and biotin were blocked with Avidin/Biotin blocking kit for $15 \mathrm{~min}$ each. Samples were then incubated with the secondary antibody goat anti rabbit biotin in DAPI/PBS solution at room temperature in the dark for $30 \mathrm{~min}$. Thereafter, cover slips were incubated with streptavidin-FITC in DAPI/PBS at room temperature in the dark for $30 \mathrm{~min}$. All incubation steps were followed by appropriate washing steps. The cover slips were transferred and fixed onto glass slides, and mounted in Citifluor API. The cover slips were examined by immunofluorescence microscopy using a Leica DMRXA microscope and Leica Software of Leica Microsystems (Wetzlar, Germany). At least six images were recorded per cover slip and the attached cells were counted.

Due to the high auto fluorescence of uncoated PAU1000, we also detached the cells after overnight culture and cytospotted the cells on glass slides for $5 \mathrm{~min}$ and speed of $500 \mathrm{rpm}$ using Shendon, Cytospin 3 apparatus. The staining procedure for Ki67 was the same as previously mentioned.

\subsection{Statistical Analysis}

A statistical analysis was performed by two-way ANOVA followed by Bonferroni post hoc analysis using GraphPad Prism software v4 (San Diego, California, USA). Values of $p<0.05$ were considered statistically significant. 


\section{Conclusions}

Polyacylurethanes (PAUs) degraded in vitro via combined bulk and surface erosion mechanisms. Due to a faster hydrolysable hard segment based on terephthaloyl diisocyanate and lower degree of microphase separation, PAUs degraded much faster in vitro than comparable PUs with the same polyester (PCL) soft segment. Predominant chain scission at the surface led to different surface properties of PAUs with respect to the bulk. Surface erosion and increased chain mobility at the surface resulted in the increase of both soft and hard segment crystallinity upon degradation. Generation of the polar groups upon hydrolysis and the increase of the hard segment content on the surface probably led to the increase in hydrophilicity, which further renders PAU1000 potentially cell adhesive. PAU1000 (molar mass of the oligocaprolactone soft segment $=1000 \mathrm{~g} / \mathrm{mol}$ ) can be recommended as a potential scaffold material to be used in regenerative medicine due to its optimal in vitro behavior. PAU1000 behaved as a non-toxic and blood-compatible biomaterial. In addition, PAU1000 supported adhesion and proliferation of human umbilical vein endothelial cells. Taken together, these results support the application of PAU1000 in the field of soft tissue repair as a temporary degradable scaffold.

\section{Acknowledgments}

The authors would like to acknowledge Ing. E.J. Vorenkamp (Department of Polymer Science, University of Groningen, The Netherlands) and J.A. Plantinga (Pathology and Medical Biology Department, Medical Biology Section, University Medical Centre Groningen, The Netherlands) for their indispensable technical assistance and expertise.

The authors are especially grateful to the W.J. Kolff Institute for Biomedical Engineering and Materials Science for co-funding this study.

\section{References}

1. Pêgo, A.P.; van Luyn, M.J.A.; Brouwer, L.A.; van Wachem, P.B.; Poot, A.A.; Grijpma, D.W.; Feijen, J. In vivo behavior of poly(1,3-trimethylene carbonate) and copolymers of 1,3-trimethylene carbonate with D,L-Lactide or $\varepsilon$-caprolactone: Degradation and tissue response. J. Biomed. Mater. Res. Part A 2003, 67, 1044-1054.

2. Lam, C.X.F.; Hutmacher, D.W.; Schantz, J.-T.; Woodruff, M.A.; Teoh, S.H. Evaluation of polycaprolactone scaffold degradation for 6 months in vitro and in vivo. J. Biomed. Mater. Res. Part A 2009, 90A, 906-919.

3. Bergsma, J.E.; Bruin, W.C.; Rozema, F.R.; Bos, R.R.M. Late degradation tissue response to poly(L-lactide) bone plates and screws. Biomaterials 1995, 16, 25-31.

4. Böstman, O.; Pihlajamäki, H.K.; Partio, E.K.; Rokkanen, P.U. Clinical biocompatibility and degradation of polylevolactide screws in the ankle. Clin. Orth. Rel. Res. 1995, 320, 101-109.

5. Lamba, N.M.K.; Woodhouse, K.A.; Cooper, S.L. Polyurethanes in Biomedical Applications; CRC Press: Boca Raton, FL, USA, 1998.

6. Endo, T.; Kanamaru, M.; Takata, T. Synthesis of poly(N-acylurethane)s, a new class of polyurethanes. Macromolecules 1994, 27, 3694-3697. 
7. Yabuta, M.; Urano, S. Polyacylurethane and Process for Producing the Same. Appl.: 432705, US Patent 5,556,933, 17 September 1996.

8. Heijkants, R.G.J.C.; Schwab, L.W.; van Calck, R.V.; de Groot, J.H.; Pennings, A.J.; Schouten, A.J. Extruder synthesis of a new class of polyurethanes: Polyacylurethanes based on poly( $\varepsilon$-caprolactone) oligomers. Polymer 2005, 46, 8981-8989.

9. Heijkants, R.G.J.C.; Schouten, A.J.; Schwab, L.W.; Roukes, F.V.; Pennings, A.J. Polyacylurethanes Based on Diisocyanates and Polyesterpolyols. Appl.: PCT/NL2005/000528, Patent: WO/2011/009443, 26 January 2006.

10. Lelah, M.D. and Cooper, S.L. Polurethanes in Medicine; CRC Press Inc.: Boca Raton, FL, USA, 1986.

11. Cheav, S.L.; Foussard-Blanpin, O. Edude comparée de la toxicité cyanés et/ou amides aromatiques. Ann. Pharmaceutiques Françaises 1990, 48, 23-31.

12. Akkapeddi, M.K.; Gervasi, J.A. Chain Extension of Polyethylene Terephthalate with Polyacyllactams. Appl.: 07/161927, US Patent: 4857603, 15 August 1989.

13. Kanamaru, M.; Takata, T.; Endo, T. Sequence change of poly(N-acylurethane)s based on transesterification. Macromol. Chem. Phys. 1996, 197, 1795-1804.

14. Castner, D.G.; Ratner, B.D. Biomedical surface science: Foundation to frontiers. Surface Sci. 2002, 500, 28-60.

15. Horbett, T.A. Principles underlying the role of adsorbed plasma proteins in blood interactions with foreign materials. Cardiovasc. Pathol. 1993, 2, 137S-148S.

16. Gõpferich, A. Mechanisms of polymer degradation and erosion. Biomaterials 1996, 17, 103-114.

17. von Burkersroda, F.; Schedl, L.; Gõpferich, A. Why degradable polymers undergo surface erosion or bulk erosion. Biomaterials 2002, 23, 4221-4231.

18. Heijkants, R.G.J.C. Polyurethane Scaffolds as Meniscus Reconstruction Materials. Ph.D. Thesis, University of Groningen: Groningen, The Netherlands, 2004. Available online: http://irs.ub.rug.nl/ppn/270337644 (accessed on 20 December 2004).

19. Pitt, C.G.; Gu, Z. Modification of the rates of chain cleavage of poly( $\varepsilon$-caprolactone) and related polyesters in the solid state. J. Control. Rel. 1987, 4, 283-292.

20. Antheunis, H.; van der Meer, J.-C.; de Geus, M.; Kingma, W.; Koning, C.E. Improved mathematical model for the hydrolytic degradation of aliphatic polyesters. Macromolecules 2009, 42, 2462-2471.

21. Pretsch, T.; Jakob, I.; Müller, W. Hydrolytic degradation and functional stability of a segmented shape memory poly(ester urethane). Polym. Deg. Stab. 2009, 94, 61-73.

22. Socrates, G. Infrared and Raman Characteristic Group Frequences: Tables and Charts, 3rd ed.; John Wiley \& sons, Inc.: Hoboken, NJ, USA, 2011.

23. Lee, H.S.; Wang, Y.K.; Hsu, S.L. Spectroscopic analysis of phase separation behavior of model polyurethane. Macromolecules 1987, 20, 2089-2095.

24. Gogolewski, S. Selected topics in biomedical polyurethanes. A review. Colloid. Polym. Sci. 1989, 267, 757-785.

25. Sun, S.; Tang, H.; Wu, P. Interpretation of carbonyl band splitting phenomenon of a novel thermotropic liquid crystalline polymer without conventional mesogens: Combination method of spectral analysis and molecular simulation. J. Phys. Chem. B 2010, 114, 3439-3448. 
26. Heijkants, R.G.J.C.; van Calck, R.V.; van Tienen, T.G.; de Groot, J.H.; Buma, P.; Pennings, A.J.; Veth, R.P.H.; Schouten, A.J. Uncatalyzed synthesis, thermal and mechanical properties of polyurethanes based on $\operatorname{poly}(\varepsilon$-caprolactone) and 1,4-butanediisocyanate with uniform hard segment. Biomaterials 2005, 26, 4219-4228.

27. Andersson, M.; Suska, F.; Johansson, A.; Berglin, M.; Emanuelsson, L.; Elwing, H.; Thomsen, P. Effect of molecular mobility of polymeric implants on soft tissue reactions: An in vivo study in rats. J. Biomed. Mater. Res. 2008, 84A, 652-660.

28. Grasel, T.G.; Cooper, S.L. Surface properties and blood compatibility of polyurethaneureas. Biomaterials 1986, 7, 315-328.

29. Kim, S.H.; Ha, H.J.; Ko, Y.K.; Yoon, S.J. Correlation of proliferation, morphology, and biological responses of fibroblasts on the LDPE with different surface wettability. J. Biomater. Sci. Polym. Edn. 2007, 18, 609-622.

30. Lee, J.H.; Khang, G.; Lee, J.W.; Lee, H.B. Interaction of different types of cells on polymer surfaces with wettability gradient. J. Colloid. Inter. Sci. 1998, 205, 323-330.

31. Johansson, S.; Svineng, G.; Wennerberg, K.; Armulik, A.; Lohikangas, L. Fibronectin-integrin interactions. Front. Biosci. 1997, 2, 126-146.

32. Seeger, J.M.; Klingman, N. Improved endothelial cell seeding with cultured cells and fibronectin-coated grafts. J. Surg. Res. 1985, 38, 641-647.

33. Harnett, E.M.; Alderman, J.; Wood, T. The surface energy of various biomaterials coated with adhesion molecules used in cell culture. Colloid. Surface. B 2007, 55, 90-97.

34. Pompe, T.; Kobe, F.; Salchert, K.; Jørgensen, B.; Oswald, J.; Werner, C. Fibronectin anchorage to polymer substrates controls the initial phase of endothelial cell adhesion. J. Biomed. Mater. Res. A 2003, 67, 647-657.

35. Voskerician, G.; Anderson, J.M.; Ziats, M.P. High molecular weight kininogen inhibition of endothelial cell function on biomaterials. J. Biomed. Mater. Res. 2000, 51, 1-9.

36. Ito, Y.; Imanishi, Y. Blood compatibility of polyurethanes. Crit. Rev. Biocompat. 1989, 5, 45-104.

37. Lenk, T.J.; Horbett, T.A.; Ratner, B.D. Infrared spectroscopic studies of time-dependent changes in fibrinogen adsorbed to polyurethanes. Langmuir 1991, 7, 1755-1764.

38. Tsuge, O.; Itoh, T.; Tashiro, M. Studies of acyl isocyanate-IV: Synthesis of diol diisocyanates. Tetrahedron 1968, 24, 2583-2590.

39. Mulder, A.B.; Blom, N.R.; Smit, J.W.; Ruiters, M.H.J.; van der Meer, J.; Halie, M.R.; Boom, V.J.J. Basal tissue factor expression in endothelial cell cultures is caused by contaminating smooth muscle cells. Reduction by using chymotrypsin instead of collagenase. Thromb. Res. 1995, 80, 399-411.

(C) 2011 by the authors; licensee MDPI, Basel, Switzerland. This article is an open access article distributed under the terms and conditions of the Creative Commons Attribution license (http://creativecommons.org/licenses/by/3.0/). 